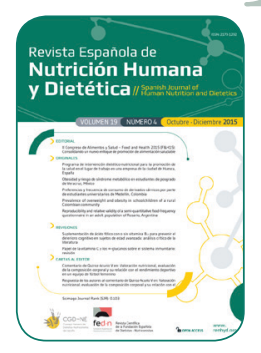

\title{
EDITORIAL
}

\section{Congreso de Alimentos y Salud - Food and Health 2015 (F\&H15): Consolidando un nuevo enfoque de promoción de alimentación saludable}

\author{
Faviola Susana Jiménez-Ramos ${ }^{a, b, c, *}$ \\ a Escuela de Nutrición y Dietética, Universidad Científica del Sur, Perú. \\ b Red Peruana de Alimentación y Nutrición, RPAN, Perú. \\ C Consejo Editorial Ejecutivo - RENHYD, España. \\ *faviolajimenez@rpan.org
}

Recibido el 8 de diciembre de 2015; aceptado el 9 de diciembre de 2015.

\section{CITA}

Jiménez-Ramos FS. II Congreso de Alimentos y Salud - Food and Health 2015 (F\&H15): Consolidando un nuevo enfoque de promoción de alimentación saludable. Rev Esp Nutr Hum Diet. 2015; 19(4): 187 - 188. DOI: 10.14306 / renhyd.19.4.202

Por segundo año consecutivo la Escuela de Nutrición y Dietética de la Universidad Científica del Sur (Perú) organizó el II Congreso de Alimentos y Salud - Food and Health 2015 (F\&H15), el cual se realizó los días 19 y 20 de noviembre. Esta versión estuvo dedicada al análisis de la oferta y la demanda de alimentos saludables en el Perú, enfatizando el aporte de la gastronomía como uno de los motores principales de la economía peruana, que se expresa en el llamado "Boom gastronómico peruano" y sus innumerables reconocimientos que incluyen la denominación de "Mejor destino gastronómico de Latinoamérica". Este boom ha sido ampliamente estudiado y se han logrado identificar importantes aportes de la gastronomía peruana como: ${ }^{1}$
1. La consolidación de la gastronomía como motor de desarrollo

2. Generadora de empleos

3. Proyección internacional

4. Articuladora

No obstante, el desarrollo del sector gastronómico contrasta con la compleja situación nutricional peruana en la que coexisten desnutridos y obesos ${ }^{2}$. Resulta también paradójica la asimetría numérica entre nutricionistas y cocineros, actualmente existen en el Perú 6.000 nutricionistas y se ha estimado que al año egresan 10.000 cocineros. Ante este 
contexto el congreso F\&H constituye una propuesta académica innovadora que propone una mirada sistémica de los procesos de alimentación y nutrición y su impacto en la salud nutricional.

El F\&H15 incluyó cuatro bloques, tres de ellos desarrollados a través de mesas redondas y una conferencia magistral; el primer bloque planteó la pregunta ¿Cómo promover una vida saludable?, en el cual se destacó la alimentación como uno de los principales determinantes de la salud; el segundo bloque abordó la oferta de alimentos saludables desde la perspectiva de la interrelación entre las cadenas alimentarias, gastronomía y salud; el tercer bloque estuvo dedicado a la demanda de alimentos saludables desde el enfoque de nuevas tendencias de consumo de alimentos y, finalmente, en el cuarto bloque se desarrolló una conferencia magistral sobre la nueva agenda de objetivos de desarrollo sostenible.

Al término de cada bloque se incluyeron tres conferencias/ exhibiciones de alimentos: la primera conferencia estuvo dedicada al tema de panificación saludable ${ }^{3}$; la segunda a la promoción del consumo de huevo ${ }^{4}$ y la tercera a la promoción del consumo de productos hidrobiológicos 5 . Al igual que en la primera versión al concluir el evento se realizó la lectura de las conclusiones del workshop de promoción de la salud y workshop de nutrición pública.

Finalmente expreso mi reconocimiento y agradecimiento a las personas e instituciones que han permitido consolidar el $\mathrm{F} \& \mathrm{H}$ como un espacio académico que permite la actualización, generación de redes de trabajo, concretar alianzas estratégicas, promover la generación de evidencias científicas y posicionar al nutricionista como líder en la promoción de salud nutricional; explícitamente mi agradecimiento a la Organización Panamericana de la Salud (OPS), Instituto Tecnológico de la Producción del Ministerio de la Producción Perú, Sociedad Peruana de Promoción de la Salud (PROMSA), Red Peruana de Alimentación y Nutrición (RPAN), Escuela Nova, Revista Industria Alimentaria Perú, Municipalidad de Miraflores y por segundo año consecutivo a la Revista Española de Nutrición y Dietética (RENHyD), revista líder en el campo de la nutrición humana y dietética.

\section{Ficheros adicionales}

Workshop Promoción de la Salud: acta de conclusiones:

http://renhyd.org/index.php/renhyd/article/ downloadSuppFile/202/77

Workshop Nutrición Pública: acta de conclusiones:

http://renhyd.org/index.php/renhyd/article/ downloadSuppFile/202/78

El resumen del encuentro científico se puede descargar en:http://www.cientifica.edu.pe/data/ archivos/20151209102510 BROCHUREF\&H15.pdf

\section{BIBLIOGRAFÍA}

1. APEGA. Asociación Peruana de Gastronomía. Gastronomía Peruana al 2021. Lineamientos para un programa de desarrollo de la gastronomía en el marco del bicentenario. 2a Edición. Lima. 2012.

2. Instituto Nacional de Estadística e Informática. Resultados de la Encuesta Demográfica y de Salud Familiar (ENDES). Lima. 2014.

3. Revista Industria Alimentaria. Industria de la Panificación. Número 17. Lima - Perú. 2012.

4. Salvador $E$, Ibarra A, Jiménez $F$, Avilés $M$, Cano $E$. El huevo de granja y su contribución a la seguridad alimentaria, nutricional y salud del consumidor. MAP. La revista del mundo avicultor y porcicultor. http://www.maplarevista.pe/2014/10/el-huevode-granjay-su-contribucion-la.html

5. Pazos M, Gonzales G, Requena F, Vera N. Manual de alimentación "Consume pescado, es mejor". Instituto Tecnológico Pesquero del Perú. Ministerio de la Producción. 1a Edición. Lima. 2010. 